\title{
The effects of organic $v$. conventional diets on immune variables in rats
}

\author{
A. M. Baranska ${ }^{1}$, E. Rembialkowska ${ }^{2}$, E. Hallmann ${ }^{2}$, L. Lueck ${ }^{3}$, J. M. Cooper ${ }^{3}$ and C. Leifert ${ }^{3}$ \\ ${ }^{1}$ Department of Animal Physiology, Faculty of Biology, University of Warsaw, Miecznikowa 1, 02-096 Warsaw, Poland, \\ ${ }^{2}$ Division of Organic Foodstuffs, Faculty of Human Nutrition and Consumer Sciences, Warsaw Agricultural University, \\ Nowoursynowska 159C, 02-776 Warsaw, Poland and ${ }^{3}$ Nafferton Ecological Farming Group (NEFG), University of \\ Newcastle, Nafferton Farm, Stocksfield NE43 7XD, UK
}

\begin{abstract}
Organic food production is gaining increasing interest worldwide. In Europe the regulations for organic crop cultivation prohibit the use of chemosynthetic pesticides, mineral fertilizers, growth promoters and genetic engineering or GM organisms. Despite a small number of studies comparing potential effects of organic and conventional foodstuffs consumption on human and animal health, there are few reports of in vitro immunosuppressive or no effects of the presence of chemical contaminants (e.g. pesticides) in conventional food ${ }^{(1)}$. The aim of the present study was, therefore, to assess the effect of diets, based on organically, low-input and conventionally-grown crops on selected immune variables of rats in an experimental feeding trial. Experimental diets were prepared according to the nutritional recommendations for rats as pellets containing dried wheat, potatoes, carrots and onions and enriched with lactoalbumin, casein, rapeseed oil, minerals and vitamins, and were characterized by the content of total flavonoids, polyphenols, $\beta$-carotene and lutein, pesticides, and antioxidant activity $^{(2)}$. Adult male and female Wistar rats were kept for 3 weeks under controlled conditions with free access to water and experimental feeds (without pesticides and mineral fertilizers, ORG-ORG; without pesticides, ORG-CV; without mineral fertilizers, CV-ORG; with pesticides and mineral fertilizers, CV-CV; control group fed Labofeed (Andrzej Morawski Feed Production Plant, Kcynia, Poland), LF), and paired and bred. Twelve young males from each experimental group were fed each of the diets for 3 and 12 weeks. Animals were killed with Thiopental overdose, blood was collected from the heart and used for haematological analysis, spleens were isolated aseptically and used immediately for in vitro studies of lymphocyte proliferation.

There were no significant differences in haematological variables of rats from all dietary groups. The effect of experimental diets on spontaneous lymphocyte proliferation was similar after 3 and 12 weeks of feeding; however, the differences were significant only after 12 weeks (ORG-ORG $v$. CV-CV, ORG-ORG $v$. ORG-CV, CV-ORG $v$. ORG-CV, CV-ORG $v$. CV-CV; $P<0.001$ ). A higher proliferation was found in rats on both diets based on products from farming systems without the use of mineral fertilizers (ORG-ORG and CV-ORG) and lower proliferation in ORG-CV and CV-CV dietary groups. These results correspond with the flavonoids and polyphenols content found in the experimental feeds. The mitogen-stimulated proliferation index was lower in ORG-ORG and CV-ORG groups. It can only be speculated whether the alteration in spontaneous lymphocyte proliferation can result from immunostimulatory effect of flavonoids and polyphenols found in feed or from the immunosuppressive influence of some dietary components in products cultivated with mineral fertilizers. Further studies are in progress.
\end{abstract}

The study was supported under the Quality Low Input Food Project: FOOD-CT-2003-506358.

1. Baranska A \& Skwarlo-Sonta K (2006) Pol J Food Nutr Sci 56, 13-16.

2. Rembialkowska E, Hallmann E, Rusaczonek A, Bennett RN, Brandt K, Lueck L \& Leifert C (2007) In Proceedings of 3rd International Congress of the European Project Quality Low Input Food $(Q L I F)$, pp. 112-117 [U Niggli, C Leifert, T Alföldi, L Lück and H Willer, editors]. Frankfurt, Germany: Research Institute of Organic Agriculture. 